\title{
The Germinative Preponderance-Sebaceous Epithelioma
}

ISSN: 2689-2707

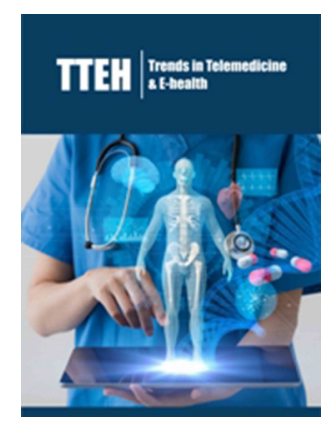

*Corresponding author: Anubha Bajaj, Histopathologist, India

Submission: 踾 August 23, 2019

Published: 洞August 30, 2019

Volume 1 - Issue 5

How to cite this article: Anubha Bajaj. The Germinative Preponderance-Sebaceous Epithelioma. Trends Telemed E-Health 1(5). TTEH. 000524. 2019.

DOI: 10.31031/TTEH.2019.01.000524

Copyright@ Anubha Bajaj, This article is distributed under the terms of the Creative Commons Attribution 4.0 International License, which permits unrestricted use and redistribution provided that the original author and source are credited.

\author{
Anubha Bajaj* \\ Department of Pathology, India
}

\begin{abstract}
Sebaceous epithelioma is a benign, exceptional, distinctive neoplasm comprised of basaloid cells and mature sebocytes. Although a sporadic neoplasm, Muir-Torre syndrome can be frequently associated with sebaceous epithelioma. Benign sebaceous epithelioma, additionally termed as sebaceoma is indicative of sebaceous neoplasm exceeding $>50 \%$ basaloid cell component and demonstrates yellow papules, nodules or plaques predominating on sites of enhanced sebaceous glands. Dermoscopy delineates yellow-tinged, granular articulations with fine, curvilinear capillaries and vascular formations. Sebaceoma incorporates an admixture of undifferentiated basaloid cells and differentiated sebaceous cells. Basaloid cells enunciate a cytoplasmic vacuolation and sebaceous cells are clear and lipid rich. Histological variants of sebaceoma include carcinoid- like, sinusoidal, reticulated, cribriform, rippled and sebaceoma with Verocay bodylike features. Immune reactivity to adipophilin, epithelial membrane antigen (EMA), cytokeratin 5(CK5), cytokeratin 6(CK6) and p40 are elucidated. Sebaceoma requires a distinction from basal cell carcinoma with sebaceous differentiation and trichoblastoma with sebaceous differentiation. Surgical resection of the neoplasm with a narrow surgical resection margin is the preferred therapeutic option.
\end{abstract}

\section{Preface}

Sebaceous epithelioma is described as a benign, exceptional, distinctive neoplasm of adnexal epithelium depicting sebaceous differentiation and can be additionally designated as sebaceoma. Sebaceous epithelioma is a benign sebaceous neoplasm comprised of basaloid cells and mature sebocytes. Muir-Torre syndrome can be frequently associated with sebaceous epithelioma, although it appears as an isolated or sporadic neoplasm. Sebaceous epithelioma can be misinterpreted as a terminology for low grade sebaceous carcinoma, basal cell carcinoma with sebaceous differentiation or sebaceous proliferations of indeterminate malignant potential [1,2].

\section{Disease Characteristics}

The face and eyelids display innumerable, modified sebaceous glands configuring as Zeis glands and Meibomian glands. Sebaceous tumors exemplifying as sebaceous adenoma, sebaceous epithelioma and sebaceous carcinoma generally are cutaneous indicators of an internal, visceral malignancy as encountered with the Muir-Torre syndrome. Sebaceoma is adopted as a nomenclature for sebaceous neoplasm exceeding $>50 \%$ basaloid cells whereas sebaceous adenoma denominates beneath $<50 \%$ basaloid cells. Sebaceoma of the eyelid appears with an incidence of $4.7 \%$ to $14.28 \%$ in the general population. Sebaceoma depicts a female predominance with an estimated female to male proportion of $4: 1$. Majority of the implicated individuals are within the sixth to ninth decade, although the tumefaction can appear at any age $[3,4]$. Sebaceoma preponderantly occurs on the head and neck, face and scalp and delineation of the aforesaid neoplasm within sites beyond the head and neck can indicate a concurrence with Muir-Torre syndrome. Thus, extensive evaluation is required to exclude concomitant systemic disease and neoplasia. However, sebaceoma can be sporadic and solitary and lack a concordance with an internal, visceral malignancy or Muir-Torre syndrome. The lesion is devoid of concurrent regional lymph node enlargement $[3,4]$.

\section{Clinical Elucidation}

The essentially benign sebaceous epithelioma, additionally termed as sebaceoma, demonstrates yellow papules, nodules or plaques which are predominant in areas of enhanced quantification of sebaceous glands. Painless, firm, non-tender nodules with well circumscribed 
borders and a varying dimension can appear on the eyelids or adjunctive locations. Yellow to orange or flesh colored papules, nodules or tumefaction can be typically elucidated in sebaceoma. Yellowish white occipital nodules with a smooth extraneous surface and magnitude in millimeters can also be enunciated. A thorough clinical investigation is necessitated in order to exclude a MuirTorre syndrome or emergence of a concomitant internal, visceral malignancy. Dermoscopic evaluation demonstrates yellow-tinged, granular articulations with accompanying finely delineated, curvilinear capillaries and vascular arrangements-a feature recapitulated in sebaceous carcinoma $[3,5]$.

\section{Histological Elucidation}

Sebaceoma typically represents as a solitary, well circumscribed, frequently yellow nodule or a poorly delineated plaque. Gross examination delineates grainy, yellowish, oily material accumulated within segregated locules. Morphological enunciation of the adnexal neoplasm incorporates an admixture of undifferentiated basaloid cells and differentiated sebaceous cells. The tumefaction is predominantly comprised of basaloid cells and loci of sebaceous differentiation with mature sebaceous cells. Basaloid cells can enunciate a cytoplasmic vacuolation and sebaceous cells are clear and lipid-rich [4,5]. Superimposed stratified squamous epithelium can depict mild induration and a prominence of vasculature. Mitotic activity is inconspicuous, and the neoplasm is devoid of stromal invasion. Dermal nodules comprised of a cellular component with irregular outline are enunciated with varying degrees of incrimination of superimposed stratified squamous epithelium. As the tumefaction is constituted of undifferentiated basaloid cells and mature, differentiated sebocytes, the neoplasm is configured by a greater $(>50 \%)$ proportion of basaloid cells. Morphological exemplification of a mammoth, intradermal tumor with cord- like structures and tumor cell nests of undifferentiated basaloid cells can also be demonstrated. The cellular aggregates appear parallel along the intervening interstitial septa in order to configure a predominantly trabecular pattern [5,6]. Emergence of miniature keratocysts within the papillary dermis or upper reticular dermis can be delineated. Minimal quantities of lipid- laden, vacuolated cells are exhibited within the tumor cell aggregates. Mitosis is infrequent and appears with the ratio of beneath $<1$ mitotic figure per 10 high power fields. Basaloid or germinative cells are accompanied with cells of sebaceous differentiation. However, germinative cells usually outnumber mature sebocytes, a feature which distinguishes sebaceoma from sebaceous adenoma. The neoplasm is generally surrounded by a sclerotic, fibrous tissue stroma. Histological variants of sebaceoma are enumerable such as carcinoid- like, sinusoidal, reticulated, cribriform, rippled and sebaceomas with Verocay body-like features [3,4]. Rippled variant of sebaceoma can exhibit a monomorphic articulation of miniature, cigar shaped, basaloid cells arranged in a parallel, linear pattern with an absence of septa betwixt rows of basaloid cells. Sinusoidal and carcinoid-like tumor evolution within a sebaceous neoplasm can be misinterpreted as a malignant transformation. Admixture of trabecular pattern and ribbon- like arrangements can be delineated. Trabecular arrangements can be enunciated singularly and entirely with a specific lesion. Variants of sebaceoma with keratoacanthomalike features or preponderant cystic modifications usually indicate the concurrence of a Muir-Torre syndrome. Sebaceomas with tumor architecture akin to carcinoid-like pattern can be particularly elucidated in malignant sebaceous carcinoma $[5,6]$.

\section{Immune Histochemical Elucidation}

A proliferative activity beneath $<10 \%$ on immune staining with diamino-benizidine with an intense, peripheral immune reactivity is exemplified. Proliferative activity of the tumefaction can also be assessed with Ki-67 (MIB-1) index which appears below $<10 \%$. Regular sebaceous glands are reactive to p21WAF1 immune antibodies, particularly within the differentiating fraction of sebaceous glands. Aforesaid compartment depicts a distinct configuration from the peripheral cellular cycle composed of Ki67 reactive basaloid cells. Proliferative index as evaluated with MIB-1 can appear at an estimated 2\% [3,4]. Sebaceoma cogitates an identical distribution of immune markers as that of normal sebaceous glands although the proliferative, cellular segment is expanded. Immune reactivity to adipophilin and epithelial membrane antigen (EMA) is delineated in zones of lipid- rich, vacuolated tumor cells. Immune reactivity to cytokeratin 5 (CK5) and cytokeratin 6 (CK6) along with p40 are elucidated in cordlike nests and vacuolated tumor cells. Immune reactive p40 can be enunciated in sebaceous carcinoma along with basal or germinative layer of sebaceous epithelium. Sebaceous cells nonreactive to adipophilin although immune reactive to p40 can represent an immature basaloid or germinative layer of sebaceous epithelium, in concordance with a typical, benign sebaceoma $[7,8]$. Immune reactive cytokeratin 5 and 6 (CK5/6) appears on account of the singularly unique trabeculae, configured by basaloid cells. Immune reactivity for epithelial membrane antigen (EMA), D240 and nuclear staining for Factor XIII a is exemplified. However, sebaceoma is nonimmune reactive for BerEp4 and S100 protein $[3,4]$.

\section{Genetic Elucidation}

Muir-Torre syndrome is contemplated as phenotypic variant of hereditary non-polyposis colorectal carcinoma syndrome (HNPCC) or Lynch syndrome. Aforesaid genomic manifestation is engendered by germline mutations occurring within one allele of DNA mismatch repair genes cogitated with MLH1, MSH2, MSH6 and PMS2. Muir-Torre syndrome is frequently accompanied with sebaceous neoplasia, chiefly sebaceous adenoma along with colorectal malignancies, genito-urinary and adjunctive visceral adenocarcinoma. Chromosomal mutations of MSH2 gene are frequently delineated in Muir-Torre syndrome $[7,8]$.

Suspected emergence of Muir-Torre, as indicated by a personal or family history, discernment of genito-urinary or breast malignancies or an elderly subject with numerous sebaceous neoplasm distant from locations within the head and neck, can be benefitted with extensive immune- histochemical evaluation of mismatch repair genes or assessment of microsatellite instability. Deficient enunciation of MLH1 on account of germline mutations 
or a somatic hyper-methylation cannot be discerned on cogent immune reactions and a few germline, missense mutations can be misinterpreted as normal immune reactivity as antigenically complete, nonfunctional proteins can be generated $[8,9]$. Microsatellite instability (MSI) assay as performed on formalin fixed tissue is sensitive and appropriate for ascertaining individuals with germline mismatch repair (MMR) genetic derangements, rather than employing immune-histochemistry. Prognostic outcomes are determined as MSI-H with enhanced proportion of microsatellite instability and MSI-L delineating minimal quantities of microsatellite instability (Figure 1-12).

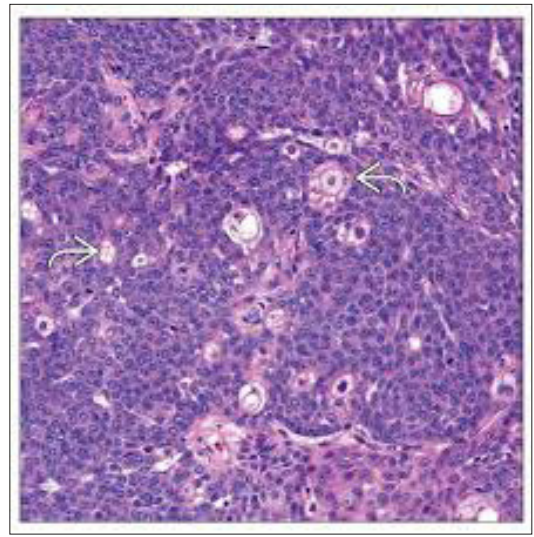

Figure 1: Sebaceoma with lobules of sebocytes and abundant, peripheral basaloid cells [11].

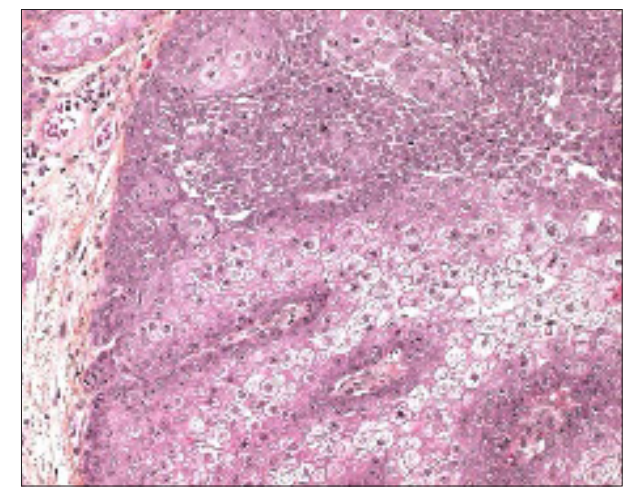

Figure 2: Sebaceoma with lobulated, lipid-rich sebaceous cells and a perimeter of undifferentiated basaloid cells [12].

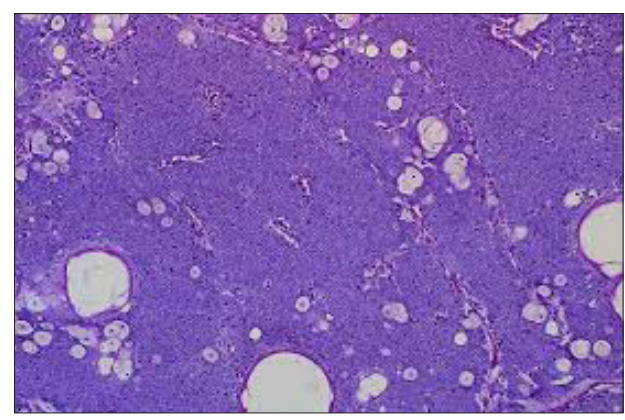

Figure 3: Sebaceoma with numerous aggregates of basaloid cells and interspersed sebocytes [13].

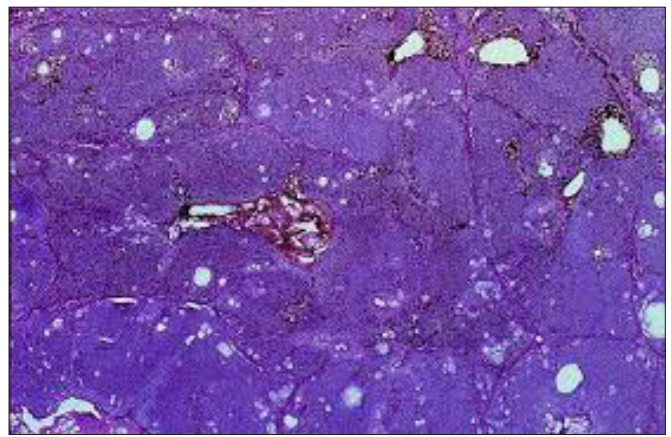

Figure 4: Sebaceoma with nests and clusters of undifferentiated basaloid cells and tiny aggregates of sebaceous cells [13].

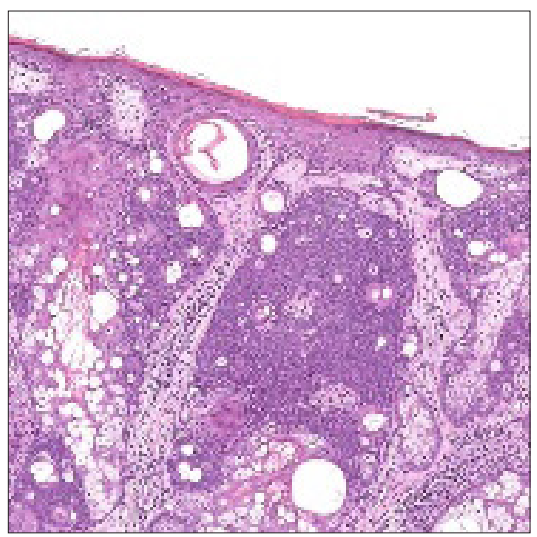

Figure 5: Sebaceoma with several, enlarged sebaceous cell clusters with a border of basaloid cells and a superimposed stratified squamous epithelium [14].

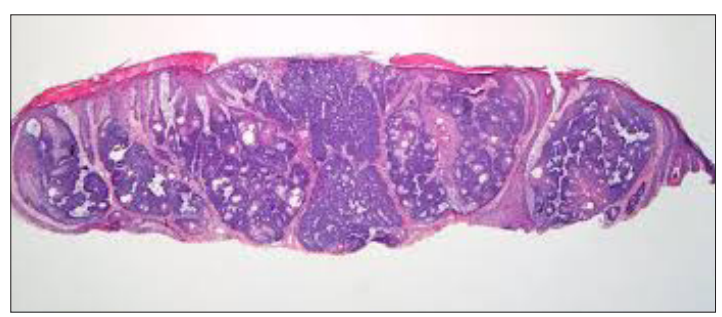

Figure 6: Sebaceoma with a dermal exponent of basaloid cell aggregates, dispersed sebaceous cells and superficial squamous epithelium [15].

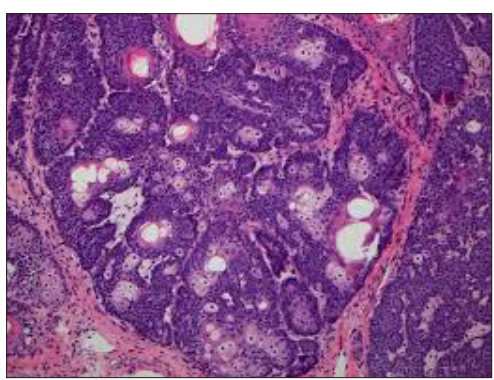

Figure 7: Sebaceoma with lobulated, dermal aggregates of undifferentiated basaloid cells and intermingles sebocytes [15]. 


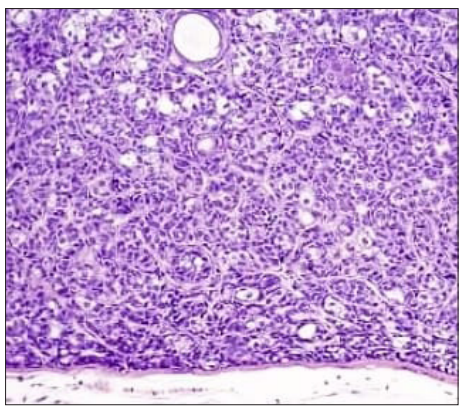

Figure 8: Sebaceoma with a commingling of mature sebocytes and peripheral basaloid cells [16].

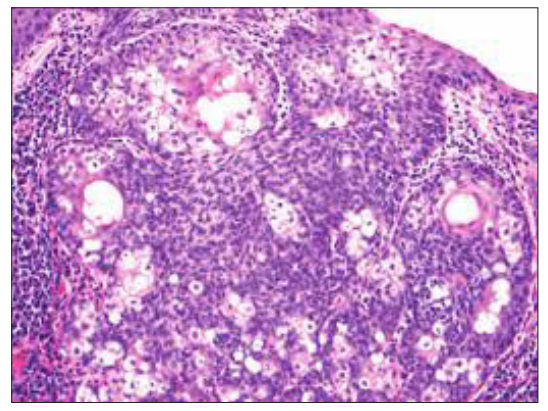

Figure 9: Sebaceoma with multi-lobular sebaceous cells and a border of undifferentiated basaloid cells [17].

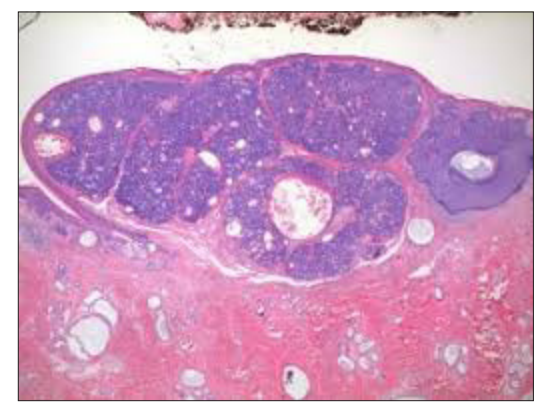

Figure 10: Sebaceoma with a dermal collusion of basaloid cell lobules configuring in the periphery and intermixed, lipid-rich, vacuolated sebocytes [18].

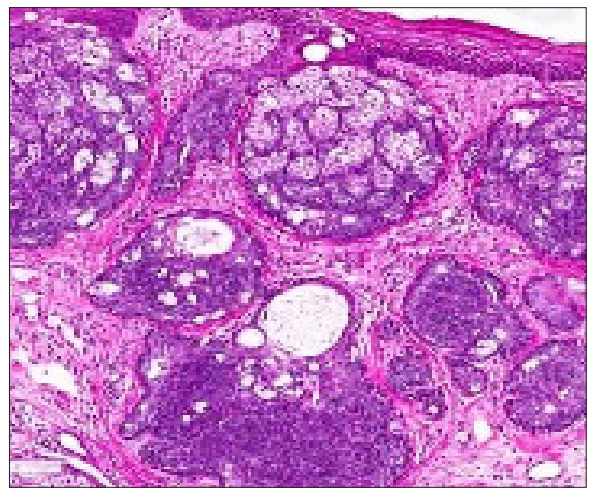

Figure 11: Sebaceoma with several nests of peripheral basaloid cells and centric sebaceous cells with a superficial stratified squamous epithelial lining [19].

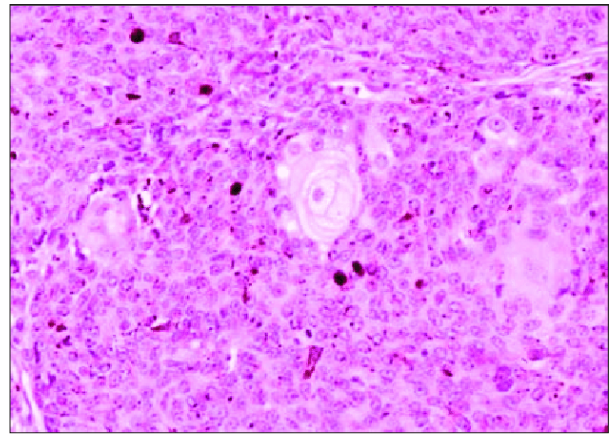

Figure 12: Sebaceoma with nodules of vacuolated sebaceous cells and a peripheral aggregation of primitive basaloid cells [20].

Evaluation of germline mutations can be adapted as blood leukocyte genomic sequencing, a modality which is optimal in the recognition of germline mutations within the MLH1, MSH2, MSH6 and PMS2 genes. Instances devoid of germline mutations can occur, although the neoplasm can depict accompanying mismatch repair deficiencies along with involvement of associated mismatch repair proteins. Somatic mutations or hyper-methylation of the promoter regions can concur. Sebaceomas can occur as sporadic neoplasm with an absence of concurrent Muir-Torre syndrome [8,9]. Specific genomic features can quantify the prognosis of sebaceoma. Absence of nuclear staining for MLH1, MSH2, MSH6 or PMS2 is indicative of microsatellite instability and favours the ascertainment of MuirTorre syndrome. Genomic mutations of MLH1 and MSH2 genes are cogent in engendering numerous instances of phenotypic microsatellite instability. Deficiency of MSH2 and MSH6 is concomitant on account of the configuration of a heterodimer mismatch repair (MMR) recognition factor generated with specific protein products of MSH2 and MSH6. MSH6 is genetically unstable in the absence of MSH2 and is rapidly deteriorated $[7,9]$.

\section{Differential Diagnosis}

Sebaceoma requires a distinction from basal cell carcinoma with sebaceous differentiation. Basal cell carcinoma depicts a predominant peripheral palisading, configuration of clefts surrounding the tumor lobules and incidental sebaceous differentiation. Basal cell carcinoma is immune reactive for BerEP4 whereas a sebaceoma is non-reactive. Sebaceous adenoma is composed of basaloid cells expressed beneath $<50 \%$, in contrast to a sebaceoma which demonstrates above $>50 \%$ of basaloid, germinative cells $[4,5]$. Sebaceous carcinoma displays analogous features of malignancy such as nuclear pleomorphism, nucleolar prominence, an infiltrative pattern of tumor evolution and an enhanced mitotic activity, in contrast to a sebaceoma. Trichoblastoma with sebaceous differentiation can also be misinterpreted as a sebaceoma and requires distinction. However, trichoblastoma delineates distinct foci of hair differentiation and papillary mesenchymal bodies $[4,5]$.

\section{Therapeutic Options}

Surgical excision of the neoplasm is the preferential therapy. Surgical resection of the nodule with a narrow surgical margin is 
optimally recommended. Reconstructive surgery of the implicated site can be adopted for a superior cosmetic result and to reduce the probability of malignant transformation. Thus, on account of possible malignant transformation within a sebaceous epithelioma and potential, localized reoccurrence, surgical extermination of the tumor with reconstructive surgery is a preferred therapeutic option $[9,10]$. Upon efficacious diagnosis, further treatment is expendable. However, a comprehensive surgical excision is optimally performed in instances of a partial biopsy or suspicion of a basal cell carcinoma with sebaceous differentiation or sebaceous carcinoma [11-20].

\section{References}

1. Torre D (1968) Multiple sebaceous tumors. Arch Dermatol 98(5): 549551.

2. Mehregan AH (1985) The origin of adnexal tumors of the skin: A viewpoint. J Cutan Pathol 12(6): 459-467.

3. Kazakov DV, Kutzner H, Rütten A, Mukensnabl P, Michal M (2005) Carcinoid like patterns in sebaceous neoplasm: Another distinctive, previously unrecognized pattern in extraocular sebaceous carcinoma and sebaceoma. Am J Dermatopathol 27(3): 195-203.

4. Misago N, Mihara I, Ansai S, Narisawa Y (2002) Sebaceoma and related neoplasm with sebaceous differentiation: A clinico-pathologic study of 30 cases. Am J Dermatopathol 24(4): 294-304.

5. Suzuki S, Sugita K, Shiomi T, Yamamoto O (2018) Benign sebaceous epithelioma with carcinoid like trabecular cell arrangement. Yonago Acta Medica 61(1): 79-81.
6. Ahuja S, Rao VA, Kumar P, Rajesh N, Srinivasan R (2014) Sebaceoma of the conjunctiva presenting as a chalazion. Indian J Dermatol 59(5): 521522.

7. Stewart CL, Novoa RA (2015) Sebaceous Epithelioma. In Lever's Histopathology of the Skin (11 $11^{\text {th }}$ edn), Philadelphia: Wolters Kluwer, pp. 1067-1068.

8. Ansai S, Kimura T (2009) Rippled pattern sebaceoma: A clinocopathological study. Am J Dermapathol 31(4): 364-366.

9. Kazakov DV, McKee P (2012) Sebaceoma. In Cutaneous Adnexal Tumors. Philadelphia: Wolters Kluwer, Netherlands, pp. 349-358.

10. Jain D, Mathur SR, Sharma MC, Iyer VK (2015) Cytomorphology of sebaceous carcinoma with analysis of p40 antibody expression. Diagnostic Cytopathology 43(6): 456-461.

11. Image 1 Courtesy: Basic medical key

12. Image 2 Courtesy: Humpath.com

13. Image 3 and 4 Courtesy: University of Pennsylvania

14. Image 5 Courtesy: Twitter

15. Image 6 and 7 Courtesy: Dermatology Advisor

16. Image 8 Courtesy: Science direct

17. Image 9 Courtesy: Wiley online library

18. Image 10 Courtesy: Dermnet NZ

19. Image 11 Courtesy: Webpathology.com

20. Image 12 Courtesy: Research Gate

For possible submissions Click below: 